\title{
Dynamic Load Rejection Control Method for Flexible Space Manipulator
}

\author{
Sun Lingzhen ${ }^{1{ }^{*}}$, Ye Shuo ${ }^{2}$, Chen Yunsheng ${ }^{1}$ \\ ${ }^{1}$ Guangzhou Huali Science and Technology Vocational College, Guangzhou, China \\ ${ }^{2}$ Huali College Guangdong University of Technology, Guangdong Guangzhou, China \\ *Corresponding author e-mail: sunlingzhen1983@163.com
}

Keywords: Flexible Space Manipulator, Control Method

\begin{abstract}
The flexible space manipulator is disturbed by the dynamic load in the position servo process, which easily leads to the unknown load distortion at the end of the manipulator, and the robustness of the flexible space manipulator control is not good. In order to improve the robustness of dynamic load rejection control for flexible space manipulator, a dynamic load rejection control method for flexible space manipulator based on load mass parameter identification is proposed. The joint flexible identification system is used to collect the position servo correlation parameters of the flexible space manipulator, and the position servo constraint parameter model of the flexible space manipulator is constructed. The terminal load mass parameter identification method is used to adjust the position error of the flexible space manipulator and the local linearization parameter fusion method is used to correct the control error of the space manipulator to improve the steady-state control performance of the flexible space manipulator. The steady state error is reduced and the on-line identification ability of unknown load parameters of space manipulator is improved. The simulation results show that the proposed method has the advantages of small error and good stability in dynamic load rejection control of flexible space manipulator, and has good performance of inertial parameter identification in orbit.
\end{abstract}

\section{Introduction}

With the development of artificial intelligence control technology, a large number of robots and manipulators are used in industrial operations and human life production. Flexible space manipulator is a new type of artificial intelligence space manipulator device. Space manipulator is an important part of orbiting spacecraft. Space manipulator is widely used in target acquisition, structural assembly and parameter identification in orbit. The space manipulator has the characteristics of light mass and high $\operatorname{span}^{[1]}$. The vibration of the structure is very obvious. For example, the space shuttle remote control arm system in the United States has to spend 20 to 40 seconds waiting for the vibration of the manipulator to stop after each operation. Especially when the manipulator captures a non-cooperative target with unknown attributes, the uncertainty of the system parameters will affect the control system. In this case, the parameters of unknown targets and the parameters of the manipulator after capture can be accurately obtained by using the on-orbit 
identification method, which can not only provide an important reference for the modification of the attitude or vibration controller ${ }^{[2]}$. It can also effectively detect the operating state of the system and ensure the normal operation of the spacecraft. The flexible space manipulator is disturbed by the dynamic load during the position servo process, which can easily lead to unknown load distortion at the end of the manipulator. It is of great significance to study the dynamic load rejection control method for flexible space manipulator.

Aiming at the identification of inertial parameters and dynamic load rejection control of the manipulator itself and the terminal load, extensive research has been carried out at present ${ }^{[3]}$. In reference ${ }^{[4]}$, a method for the identification of inertial parameters in orbit based on spacecraft manipulator is proposed, which realizes the identification of inertial parameters of spacecraft by the wide range motion of the manipulator. In reference ${ }^{[5]}$, according to the principle of conservation of momentum, the mass characteristic parameters of unknown targets are obtained from the simultaneous momentum increment equations in the process of grasping unknown targets by space robots. In reference ${ }^{[6]}$, an algebraic identification method is proposed, and an on-line identification method for the mass parameters of the end load of the manipulator under gravity environment is proposed. In reference ${ }^{[7]}$, aiming at the problem of catching unknown loads for space manipulators, the identification and disturbance control of load characteristic parameters are carried out by using joint torque and tactile sensing information. In most of the above studies, the manipulator is considered as a rigid body, and the influence of flexible vibration is ignored, and because of the limited excitation source in the space environment, many parameter identification methods are not applicable.

In this paper, we mainly focus on the parameter identification of the manipulator under a fixed configuration, so in the process of capturing the target, the parameters of the system model are only affected by the change of the terminal mass. Therefore, the nonlinear model of the manipulator can be reduced to a linear system by using the local linearization technique. In this paper, a dynamic load rejection control method for flexible space manipulator based on load mass parameter identification is proposed. The joint flexible identification system is used to collect the position servo correlation parameters of flexible space manipulator. The position servo constraint parameter model of flexible space manipulator is constructed, the position error of flexible space manipulator is adjusted by the identification method of terminal load quality parameter, and the dynamic load immunity control is realized. Finally, the simulation experiment is carried out. The advantages of this method in improving the dynamic load rejection control capability of flexible space manipulator are demonstrated.

\section{Description of controlled object and optimization of control constraint parameters for dynamic loading system of space manipulator}

\subsection{Description of position servo controlled object of flexible space manipulator}

In order to realize the optimal control of the dynamic load system of the flexible space manipulator, the position servo parameters of the flexible space manipulator are collected by using the sensor measurement method, and the position and attitude information of the space manipulator is collected. The mechanical parameter information and ranging information of mechanical components are fused by means of inertial parameter adjustment and multi-position measurement, and the control constraint parameters are analyzed according to the measurement results. The control object model of the flexible space manipulator is established. When the controlled object model of a mechanical quilt is carried out, the following assumptions are given to simplify the handling of the configuration of the flexible space manipulator with respect to the $x_{1} O y_{1}$ plane 
symmetry. Inertial moment of flexible space manipulator in operation, the effect of disturbance torque is linearly varying, and the disturbance error is linearly variable. For the plane problem, the vibration along the arm length (axial) direction is not considered ${ }^{[8]}$. Only the vibration perpendicular to the length of the arm rod is considered, and the vibration is considered as a small deformation, and the effect of the flexibility of the joint on the vibration of the system is not considered for the time being. Based on the above assumptions, the controlled reference model of flexible space manipulator control is constructed, and the vibration displacement can be decomposed into:

$$
\begin{gathered}
\varsigma_{1}(t, s)=\left[\begin{array}{lll}
x^{T}(t) & x^{T}(t-\tau) & \dot{x}^{T}(t)
\end{array}\right]^{T} \\
\varsigma_{2}(t, s)=\left[\begin{array}{lll}
y^{T}(t) & y^{T}(t-\sigma) & \dot{y}^{T}(t)
\end{array}\right]^{T}
\end{gathered}
$$

Assuming that $\tau_{s c}$ is the hysteresis factor for dynamic load rejection control of space manipulator, $\tau_{c a}$ is the time delay transmitted to the actuator by the controller ${ }^{[9]}$. In order to further establish the controlled object model of flexible space manipulator, the following constraints are given:

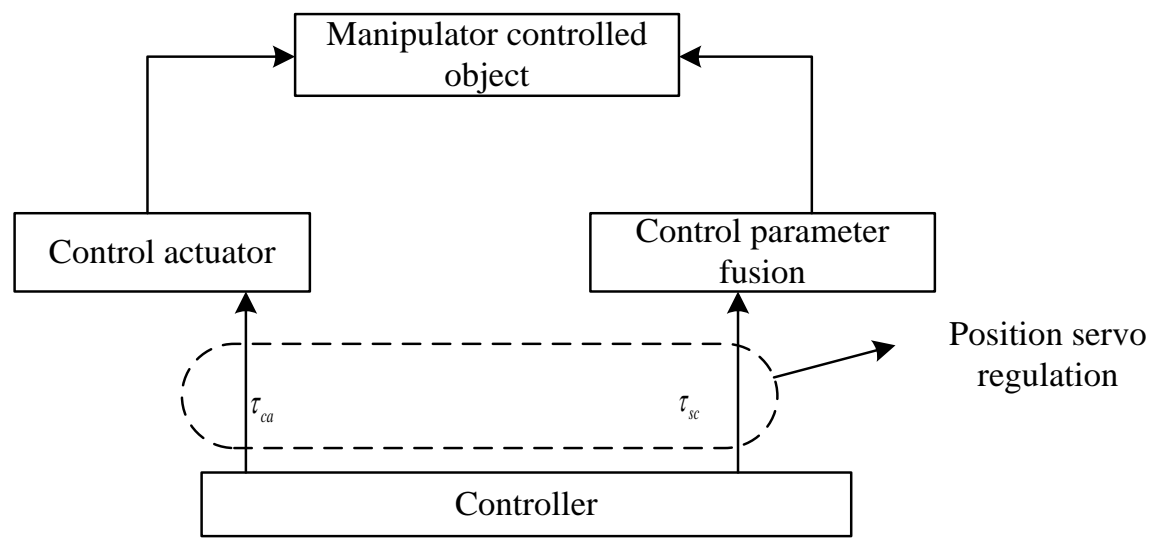

Figure 1. Controlled object model of dynamic load rejection control for flexible space manipulator.

(a) Under the action of small disturbance moment, the transmission delay $\tau_{k}$ of the flexible space manipulator is uncertain and satisfied: $\tau_{k}=\tau_{s c}+\tau_{c a}$.

(b) The sampling period of the flexible space manipulator motion of the sensitive element is $\mathrm{T}$.

(c) The control error between controller and actuator is steady.

According to the above model assumptions and conditions, the controlled object model of dynamic load rejection control for flexible space manipulator is constructed as shown in figure 1.

\subsection{Control constraint parameter optimization}

According to the control object model of flexible space manipulator constructed above, considering the state feedback control law $u(k)=\boldsymbol{K x}(k)$, when the output delay $\tau_{k}$ of flexible space manipulator is less than a sampling period, according to the vertical axis of flexible space manipulator, based on the position servo parameter correction model of (Y-axis), the parameter correlation matrix of the controlled object is obtained by using the small disturbance suppression method:

$$
\left(u, u_{t}\right) \in C_{t}\left(K, \dot{H}_{x}^{s_{c}} \times \dot{H}_{x}^{s_{c}-1}\right), u \in L_{t, x}^{d+1}\left(K \times I R^{d}\right)
$$


The methods of inertial parameter adjustment and parameter adaptive fusion are used, when the transfer function is determined[10], the feedback output control instructions of the position servo tracking system of $G_{m}(s)=G_{0}(s), t_{m}=\tau$, flexible space manipulator are as follows:

$$
H(s)+Y(s)=G_{m}(s) U(s)
$$

The dynamic load rejection control system of the flexible space manipulator forms a two-degreeof-freedom IMC-PID controller, which adaptively adjusts the control parameters and obtains the feedback information matrix of the position servo of the flexible space manipulator:

$$
\left[\begin{array}{ll}
G_{11}(s) & G_{12}(s \\
G_{21}(s & G_{22}(s
\end{array}\right]=\left[\begin{array}{cc}
\frac{1.7 e^{-30 s}}{7 s+1} & \frac{0.59 e^{-27 s}}{8 s+1} \\
\frac{-0.6 e^{-25 s}}{10 s+1} & \frac{1.5 e^{-28 s}}{9 s+1}
\end{array}\right]
$$

In the upper formula, the input control parameters of the flexible space manipulator with degrees of freedom are equivalent to the eigenvalues obtained by adjusting the parameters adaptively directly from the $G_{m}(s)$, and the nonlinear dynamics of the manipulator are established without considering the effect of joint flexibility on the system. Learn the equation and linearize at the working point ${ }^{[11]}$.

\section{Optimization of dynamic load control method}

\subsection{Control error correction of space manipulator}

The joint flexible identification system is used to collect the position servo correlation parameters of the flexible space manipulator ${ }^{[12]}$, and the position parameter acquisition model of the flexible space manipulator in the relative inertial space is presented as:

$$
\mathbf{z}(\boldsymbol{t})=\sum_{i=1}^{p} s_{i}(t) \boldsymbol{b}_{i}\left(\theta_{i}\right)+\boldsymbol{n}(t)
$$

Where:

$$
\boldsymbol{b}_{\boldsymbol{i}}\left(\theta_{i}\right)=\int_{-\pi}^{\pi} \boldsymbol{a}(\boldsymbol{\theta}) g_{i}\left(\theta-\theta_{i}\right) d \theta
$$

By using the method of inertial parameter fusion and adaptive compensation, the eigenvalues of dynamic load rejection control parameters of flexible space manipulator are decomposed, and the eigenvalues are obtained as $\lambda_{1}>\lambda_{2}>\ldots>\lambda_{j-1}>\lambda_{j}>\ldots>\lambda_{m}$, a two-degree-of-freedom control model with time-delay is constructed according to the torsion and the position servo motion coefficient of the space manipulator:

$$
\sum_{k=1}^{m}\left[\theta_{j k}(n+1)-\theta_{j k}(n)\right] q_{k}=\sum_{k=1}^{m} \eta\left[\lambda_{k}-\delta_{j}^{2}(n)\right] \theta_{j k}(n) q_{k}+\eta \sum_{k=1}^{j-1} \lambda_{k} a_{j k}(n) q_{k}
$$

The Lagrange equation is used to derive the generalized coordinates respectively ${ }^{[13]}$. The singular value of the external moment covariance matrix is decomposed into:

$$
\mathbf{R}=\mathbf{U}_{\mathrm{s}} \Lambda_{\mathrm{s}} \mathbf{U}_{\mathrm{s}}^{\mathrm{H}}+\mathbf{U}_{\mathrm{n}} \Lambda_{\mathrm{n}} \mathbf{U}_{\mathrm{n}}^{\mathrm{H}}
$$

According to the above parameter regulation model, the optimal design of the control model is carried out by using the two degree-of-freedom time-delay compensation control and the adaptive 
parametric adjustment method, which improves the global stability of the dynamic load disturbance rejection control.

\subsection{Optimal realization of dynamic load rejection control for space manipulator}

The position servo constraint parameter model of flexible space manipulator is constructed, and the position error of flexible space manipulator is adjusted by the identification method of terminal load mass parameter. The input and output relation of position parameter adjustment is obtained by designing parameter fusion filter for information fusion:

$$
x(n)+\sum_{k=1}^{p} a_{i} x(n-k)=\sum_{r=0}^{m} b_{r} u(n-r)
$$

Set $\Delta x_{k}=\alpha p_{k}, \alpha$ is the error feedback correction coefficient. The time-delay parameter adjustment and adaptive geometric calibration of dynamic load rejection control for flexible space manipulators are introduced by continuous feedback drive method. The dynamic mechanical characteristic equations of dynamic load rejection control for space manipulators are described as follows:

$$
\left\{\begin{array}{l}
W_{i}^{T}(x) X(n)=X^{T}(n) w_{j}(n) \\
a_{j}{ }^{T}(n) Y_{j-1}(n)=Y_{j-1}{ }^{T} a_{j}(n)
\end{array}\right.
$$

According to the local linearization theory, the damping characteristic equation of space manipulator dynamic load system is obtained as follows:

$$
w_{j}(n+1)=w_{j}(n)+\eta\left[y_{j}(n) X(n)-y_{j}{ }^{2}(n) w_{j}(n)\right]=w_{j}(n)+\eta\left[X(n) X^{T}(n) w_{j}(n)+X(n) X^{T}(n) Q^{T} a_{j}(n)-y_{j}{ }^{2}(n) w_{j}(n)\right]
$$

The system mass matrix coupling coefficient matrix and stiffness matrix are constructed to improve the steady-state adjusting ability of dynamic load rejection control of space manipulator. Based on the local linearization parameter fusion method, the control error correction of space manipulator is realized, and the bias analysis of space manipulator is carried out. The geometric variation relations of each overpass are obtained as follows:

$$
\begin{gathered}
x_{i}(n)=\sum_{j=1}^{M} h_{i j}(n)^{T} \boldsymbol{s}_{j}(n)+v_{i}(n) \\
y_{j}(n)=\sum_{i=1}^{P} f_{i j}(n)^{T} \boldsymbol{x}_{i}(n)
\end{gathered}
$$

Where, $h_{i j}$ represents the mechanical damping parameters of each section, and $f_{i j}$ represents the sensitivity characteristic. Thus the position servo modal parameter identification output of flexible space manipulator is obtained as:

$$
f_{i j}(n+1)=f_{i j}(n)+\mu_{M C M A} \frac{\partial J_{M C M A}(n)}{\partial f_{i j}(n)}
$$

The $\mu_{\text {MCMA }}$ represents the degree of axial position and adaptively modifies the parameters according to the number of mode shapes and rotation angles in the modeling process, combining with the method of local linearization parameter fusion to realize the control error of space manipulator. Difference correction, the output of the optimized parameters under the variable step size is as follows: 


$$
f_{i j}(n)=\left[f_{i j}^{(0)}(n), f_{i j}^{(1)}(n), \cdots, f_{i j}^{(L-1)}(n)\right]^{T}
$$

Without considering the effect of damping, the parameters of load mass at the end of the manipulator are identified. According to the above algorithm design, the error of the dynamic load rejection control model of the flexible space manipulator designed in this paper converges to zero and the control is controlled. The steady state of the process converges. The implementation of the control algorithm is shown in figure 2.

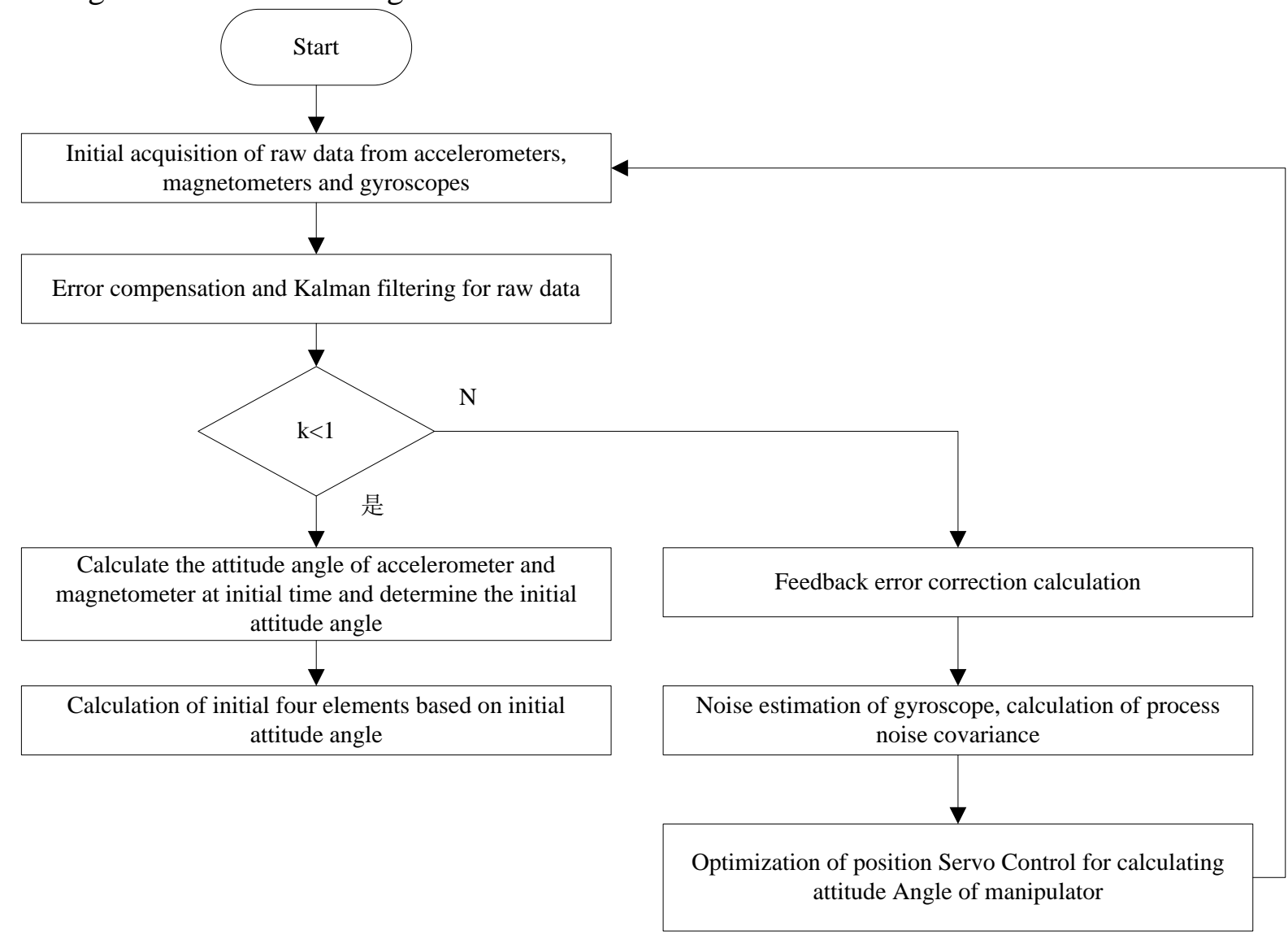

Figure 2. Flow chart of control implementation.

\section{Simulation experiment and result analysis}

In order to test the application performance of this method in the dynamic load rejection control of flexible space manipulator, the simulation experiment is carried out, and the algorithm of the simulation experiment is designed to run on the Matlab7.0 numerical simulation platform. The hardware environment of the experiment is: Tektronix TX3 True RMS MultiMeter, input flexible space manipulator position servo attitude data sampling array source expansion angle is $\Delta_{1}=5^{0}, \Delta_{2}=8^{0}$, flexible space manipulator position servo disturbance signal to noise ratio of $12 \mathrm{~dB}$, main side. With a height ratio of $22 \mathrm{~dB}$, the position servo stability control decision matrix of flexible space manipulator is constructed as follows: 


$$
D=\left[\begin{array}{cc}
2.6366 & 1.7014 \\
2 & 5 \\
0.9517 & 0.9500 \\
6429.3329 & 4748.0429 \\
48.76878 & 893.90164 \\
0.2913 & 0.2889 \\
5 & 5
\end{array}\right]
$$

The optimal solution of position servo parameters of flexible space manipulator is obtained. The results are expressed as follows: $\vartheta=(1.45,2,0.32,46.8,48.87,0.65,5.45)$.

According to the results of the above parameter calculation, the dynamic load rejection control simulation experiment of flexible space manipulator is carried out, and the dynamic load parameter identification results of flexible space manipulator are obtained as shown in Fig. 3.
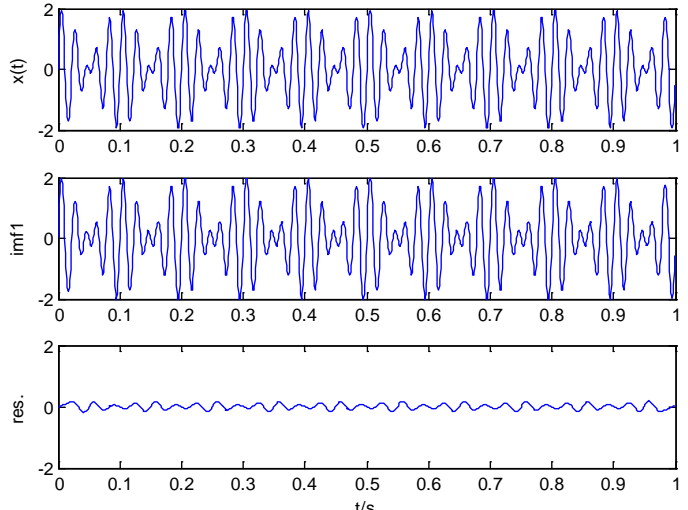

Figure 3. Identification results of parameters of space manipulator.

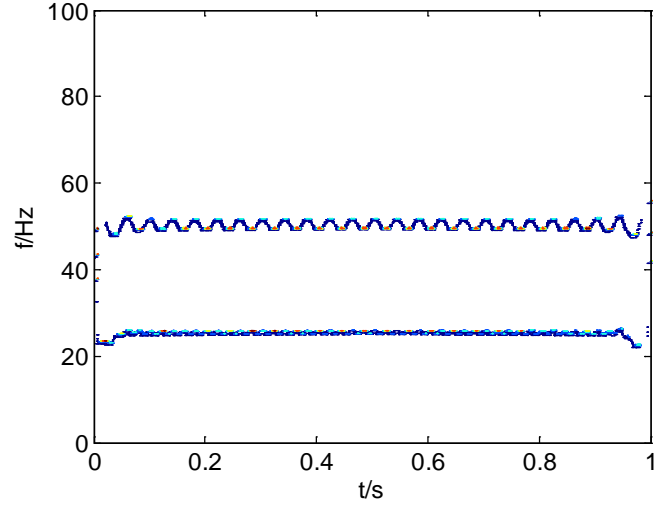

Figure 4. Performance curve of dynamic load rejection control for flexible space manipulator.

Figure 3 shows that the dynamic load rejection control of the flexible space manipulator using this method is better than that of the static tracking control of the position servo parameters of the space manipulator. In order to quantitatively analyze the method in this paper, the flexible space is carried out. The error convergence of the dynamic load rejection control of the manipulator is obtained. The desired and actual output of the dynamic load rejection control parameters of the flexible space manipulator are tested. The control performance curve is shown in figure 4.

Figure 4 shows that the control convergence of flexible space manipulator control using this method is good and the expected output is in good agreement with the actual output. The time cost of dynamic load rejection control is measured by different methods. As shown in figure 5, the time cost of dynamic load rejection control for space manipulator by this method is smaller. The average time cost of Kalman filter control is $12.34 \mathrm{~ms}$, the time cost of spatial tracking is $23.44 \mathrm{~ms}$. the time cost of this control method is $8.65 \mathrm{~ms}$. it is superior in real-time control. 


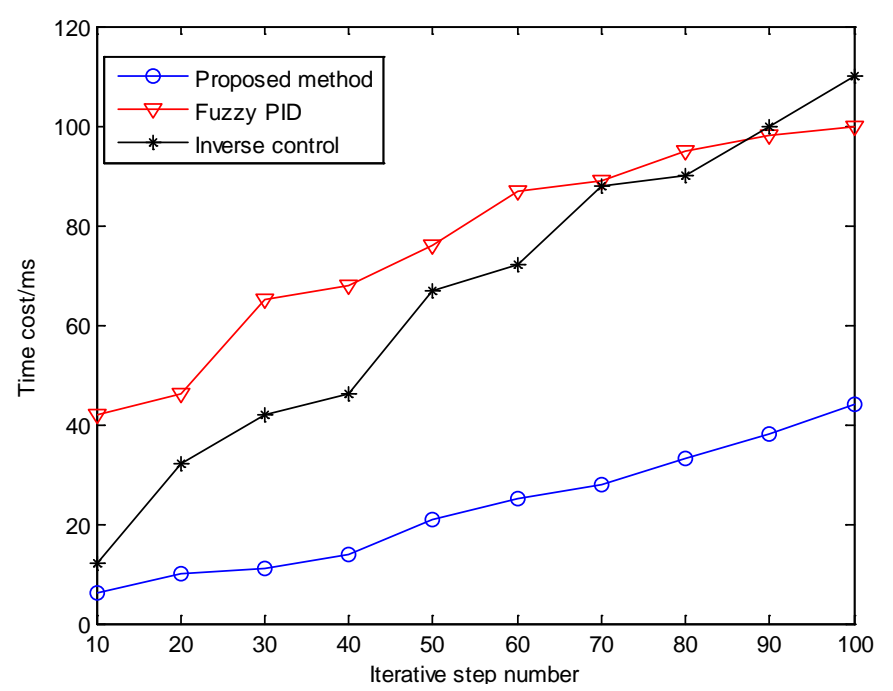

Figure 5. Comparison of Time Cost.

\section{Conclusions}

In order to improve the robustness of dynamic load rejection control of flexible space manipulator, a flexible space manipulator dynamic load rejection control method based on load mass parameter identification is proposed in this paper. The flexible joint identification system is used for flexible control. The position servo correlation parameter of the flexible space manipulator is collected, the position servo constraint parameter model of the flexible space manipulator is constructed, and the position error of the flexible space manipulator is adjusted by the identification method of the terminal load mass parameter. The control error correction of space manipulator is realized by combining local linearization parameter fusion method. The steady state control performance of flexible space manipulator is improved, the steady state error is reduced, and the online identification ability of unknown load parameters of space manipulator is improved. The simulation results show that the proposed method has the advantages of small error and good stability in dynamic load rejection control of flexible space manipulator, and has good performance of inertial parameter identification in orbit. This method has good application value in the control optimization of manipulator.

\section{References}

[1] You Y P, Zhang Y, Li C G. Force-free control for the direct teaching of robots [J]. Journal of Mechanical Engineering, 2014, 50(3):10-17.

[2] Uijlings $J R R$, van de Sande K E A, Gevers T, et al. Selective search for object recognition [J]. International Journal of Computer Vision, 2013, 104(2):154-171.

[3] ZHANG Danfeng, LI Bin, WANG Liyan. Tracking Control Method of the Centre-of-Mass Velocity for a Snake-like Robot Based on the Continuum Model [J]. ROBOT, 2017, 39(6): 829-837.

[4] LI Ke,MI Jie. Research on Mechanical and Electrical Control Algorithm of Bionic Robot Based on Variable Structure PID [J].Journal of Henan University of Engineering (Natural Science Edition), 2016,28(2):32-37.

[5] Ren Z W, Zhu Q G, Xiong R. A joint physical constraints avoidance method for inverse kinematics problem of redundant humanoid manipulator [J]. Journal of Mechanical Engineering, 2014, 50(19):58-65.

[6] JING Fengshui, YANG Chao, YANG Guodong, et al. Robot Trajectory Rectification Control Methods [J]. ROBOT, 2017, 39(3): 292-297.

[7] DU Xuedan, CAI Yinghao, LU Tao, et al. A Robotic Grasping Method Based on Deep Learning [J]. ROBOT, 2017, 39(6): 820-828,837.

[8] LIU Kai, ZHU Ji-hong, YU Bo. Longitudinal control of aircraft with thrust vectoring using robust dynamic inversion [J]. Control and Decision, 2013, 28(7): 1113-1116. 
[9] Varley $J$, Weisz $J$, Weiss $J$, et al. Generating multi-fingered robotic grasps via deep learning[C]//IEEE/RSJ International Conference on Intelligent Robots and Systems. Piscataway, USA: IEEE, 2015:4415-4420.

[10] Zeiler M D, Fergus R. Visualizing and understanding convolutional networks[C]//13th European Conference on Computer Vision. Berlin, German: Springer, 2014:818-833.

[11] Ren $S$ Q, He K M, Girshick R, et al. Faster R-CNN:Towards real-time object detection with region proposal networks[M]//Advances in Neural Information Processing Systems. Cambridge, USA: MIT Press, 2015:91-99.

[12] Lu Xinghua, Zhen Hanjian, Duan Wuxing. Fault Tolerant Controller Design for Embedded Multi Mode Control System [J]. Machinery \& Electronics, 2016, 34(4): 62-65.

[13] Liu Wei, Lu Xinghua. Research on Robust Control of Unmanned Aircraft Inertial Navigation System under Condition of Flight Imbalance [J]. Computer \& Digital Engineering, 2016, 44(12): 2380-2385. 